\title{
Challenges in the Management of Metastatic Soft Tissue Sarcomas: where are we going?
}

\author{
Luca Paoluzzi* \\ Department of Medicine, NYU Langone Medical Center, USA
}

Submission: March 24, 2018; Published: March 29, 2018

*Corresponding author: Luca Paoluzzi, Department of Medicine, NYU Langone Medical Center, 160 E $34^{\text {th }}$ Street, New York, NY 10016, Tel: +1-2127316667; Fax: +1-2127315527; Email: Luca.Paoluzzi@nyumc.org

Keywords: Soft tissue sarcoma; Next generation sequencing; Larotrectinib; Tazemetostat; Check-point inhibitors; Leiomyosarcoma; Liposarcoma; Undifferentiated sarcoma

Abbreviations: STS: Soft Tissue Sarcoma; LMS: Leiomyosarcoma; LPS: Liposarcoma; UPS: Undifferentiated Pleomorphic Sarcoma; ASPS: Alveolar Soft Part Sarcoma; PEComa: Perivascular Epithelioid Tumor; DDLPS: Dedifferentiated Liposarcoma; NGS: Next Generation Sequencing; TMB: Tumor Mutational Burden; MFS: Myxofibrosarcoma; TRK: Tropomyosin Kinase; ES: Epithelioid Sarcoma

\section{Introduction}

Soft tissues sarcomas (STS) are a heterogeneous group of diseases with more than 70 entities described in the last WHO classification [1] and 13,040 estimated new cases in the US in 2018 [2]; the most common subtypes, excluding gastrointestinal stromal tumors, are represented by leiomyosarcoma (LMS), liposarcoma (LPS) and undifferentiated/unclassified sarcomas. Four new drugs have been approved by the FDA for the treatment of metastatic STS since 2012: olaratumab in the first line setting, in combination with doxorubicin; [3] pazopanib, a tyrosine kinase inhibitorfor all subtypes but adipocytic tumors [4]; trabectedin for LMS and LPS [5]; eribulin for LPS [6] (the last three drugs were all approved for after prior chemotherapy).

During the last several years it has become progressively more clear that different subtypes of STS may respond differently to distinct treatments; one of the oldest examples in this regard comes from angiosarcoma that is known for its sensitivity to paclitaxel; additional examples include alveolar soft part sarcoma (ASPS) for its sensitivity to tyrosine kinase inhibitors and, most recently, to check point inhibitors; malignant perivascular epithelioid tumors (PEComa) that may respond to mTOR inhibition with drugs such as sirolimus or inflammatory myofibroblastic tumors to crizotinib and so on.

Immunotherapies based on check-point inhibitors are currently revolutionizing the way we treat multiple malignancies. The first demonstration of efficacy of immunotherapy in cancer actually comes from a patient with a neck sarcoma, back in
1891, who had a complete response after injection of a culture of streptococcus erysipelas [7]. Two recent prospective phase 2 studies explored the activity of checkpoint inhibitors in STS: the response rate was $18 \%(7 / 40)$ for pembrolizumab alone [8], 5\% for nivolumab alone $(2 / 38)$ and $16 \%(6 / 38)$ for nivolumab plus ipilimumab [9].

During the last few years, next generation sequencing (NGS) has shown the potential to impact the diagnosis and treatment of STS positively; a retrospective analysis of 5,635 patients worldwide included 56 different histologies; subjects have been profiled for 405 cancer-related genes in the DNA and 265 gene rearrangements in the RNA; 1165 fusions and more than 60,000 mutations were found; up to $42 \%$ of patients had some type of alterations that made them eligible for a targeted therapy in the context of a "basket" trial such as the NCI-MATCH trial [10].

Additional useful information from NGS analysis is represented by the tumor mutational burden (TMB); TMB has been associated with response to check-point inhibitors in multiple malignancies such as melanoma and non-small cell lung cancer. In an analysis of 100,000 human cancer genomes, sarcomas generally have showed relatively low TMB, but cases with high TMB have also been reported [11], this information may be of particular interest in less frequent histologies for which genomic data is very limited.

A comprehensive and integrated genomic characterization of adult STS has been recently published by the Cancer Genome 
Atlas Research Network [12]: 206 sarcomas, representing 6 major types were analyzed through a multi-platform molecular approach. Copy number changes with low mutational loads and only a few genes highly recurrently mutated were noted across all subtypes (TP53, ATRX, RB1). Generally, deletions and mutations in tumor suppressor genes were more common than amplifications and mutations in oncogenes. Specific pathway activation with distinct molecular subtypes associated with clinical outcomes were found in dedifferentiated liposarcoma (DDLPS) and soft tissue LMS; furthermore, a study of the tumor immune microenvironment through DNA methylation and mRNA profiling revealed immune cell infiltration in genomically complex DDLPS, LMS, undifferentiated pleomorphic sarcoma (UPS) and myxofibrosarcoma (MFS). Of note, objective responses to check-point inhibitors in prospective clinical trials have been reported in the aforementioned subtypes with 4/10UPS and 2/10LPS responding to pembrolizumab, 2LMS, 1MFS, 2UPS out of 38 patients responding to nivolumab + ipilimumab, 1LMS out of 38 patients responding to nivolumab alone. Additional histologies that showed responses in these trials included one angiosarcoma treated with nivolumab + ipilimumab, one ASPS treated with nivolumab alone and one synovial sarcoma who received pembrolizumab $[8,9]$.

Classically, cancer patients have been enrolled into clinical trials based on specific histologic and age criteria. Basket trials with a focus on peculiar genomic characteristics are now inducing a paradigm shift in the treatment of many cancers; rare cancers such as STS will especially benefit from this new trial design because of the obvious challenges in developing prospective studies in uncommon disease.

One of the most successful examples in this regard is the clinical development of larotrectinib, a potent and selective smallmolecule inhibitor of tropomyosin kinase (TRK) proteins. In a cohort of 55 patients with 17 different types of cancers including 18 patients with STS, larotrectinib showed objective responses in $75 \%$ of patients with 7 complete responses. Of note, two pediatric patients with locally advanced fibrosarcoma were able to undergo limb-sparing surgery with curative intent after preoperative treatment [13]. Other stories such as the development of MDM2 or CDK4 inhibitors for dedifferentiated LPS, have been less successful so far, but multiple target therapies are in clinical development. Additional treatment strategies include targeting the epigenetic cellular machinery; one example comes from the use of EZH2 inhibitors in INI1 negative tumors such as epithelioid sarcoma (ES). ES is notoriously a very aggressive and chemo-resistant STS subtype for which only surgical resection has shown to be potentially curative to date. An ongoing phase 2 multicenter trial is exploring the activity of the EZH2 inhibitor Tazemetostat in patients with INI1 negative tumors including ES; preliminary results presented at the 2017 ASCO meeting showed one partial response in ES [14]. Interestingly, one transient partial response to nivolumab and pazopanib has been noted in a patient with ES in a recent retrospective series [15].

\section{Conclusion}

In conclusion, new genomic tools and trial designs are refining the diagnosis and treatment of STS increasing the knowledge on these heterogeneous diseases and providing new hope to patients.

\section{References}

1. Fletcher CDM, Bridge JA, Hogendoorn PCW, Mertens F (2013) World Health Organization (WHO) Classification of Tumors of Soft Tissue and Bone. Pathology and Genetics. Lyon, France: IARC Press, pp. 218-220.

2. Siegel RL, Miller KD, Jemal A (2018) Cancer statistics. CA Cancer J Clin 68(1): 7-30.

3. Tap WD, Jones RL, Van Tine BA, Chmielowski B, Elias AD, et al. (2016) Olaratumab and doxorubicin versus doxorubicin alone for treatment of soft-tissue sarcoma: an open-label phase $1 \mathrm{~b}$ and randomised phase 2 trial. Lancet 388(10043): 488-497.

4. van der Graaf WT, Blay JY, Chawla SP, Kim DW, Bui-Nguyen B, et al. (2012) Pazopanib for metastatic soft-tissue sarcoma (PALETTE): a randomised, double-blind, placebo-controlled phase 3 trial. Lancet 379(9829): 1879-1886.

5. Demetri GD, Chawla SP, von Mehren M, Ritch P, Baker LH, et al. (2009) Efficacy and safety of trabectedin in patients with advanced or metastatic liposarcoma or leiomyosarcoma after failure of prior anthracyclines and ifosfamide: results of a randomized phase II study of two different schedules. J Clin Oncol 27(25): 4188-4196.

6. Schöffski P, Chawla S, Maki RG, Italiano A, Gelderblom H, et al. (2016) Eribulin versus dacarbazine in previously treated patients with advanced liposarcoma or leiomyosarcoma: a randomised, open-label, multicentre, phase 3 trial. Lancet 387(10028): 1629-1637.

7. Parish CR (2003) Cancer immunotherapy: the past, the present and the future. Immunol Cell Biol 81(2): 106-113.

8. Tawbi HA, Burgess M, Bolejack V, Van Tine BA, Schuetze SM, et al. (2017) Pembrolizumab in advanced soft-tissue sarcoma and bone sarcoma (SARC028): a multicentre, two-cohort, single-arm, openlabel, phase 2 trial. Lancet Oncol 18(11): 1493-1501.

9. D’Angelo SP, Mahoney MR, Van Tine BA, Atkins J, Milhem MM et al. (2018) Nivolumab with or without ipilimumab treatment for metastatic sarcoma (Alliance A091401): two open-label, noncomparative, randomised, phase 2 trials. Lancet Oncol 19(3): 416-426.

10. Gounder MM, Ali SM, Robinson V, Bailey M, Ferraro R, et al. (2017) Impact of next-generation sequencing (NGS) on diagnostic and therapeutic options in soft-tissue and bone sarcoma. J Clin Oncol 3 (suppl abstract 11001).

11. Chalmers ZR, Connelly CF, Fabrizio D, Gay L, Ali SM, et al. (2017) Analysis of 100,000 human cancer genomes reveals the landscape of tumor mutational burden. Genome Med 9(1): 34.

12. Cancer Genome Atlas Research Network, Cancer Genome Atlas Research Network (2017) Comprehensive and Integrated Genomic Characterization of Adult Soft Tissue Sarcomas. Cell 171(4): 950.e28965.e28.

13. Drilon A, Laetsch TW, Kummar S, DuBois SG, Lassen UN, et al. (2018) Efficacy of larotrectinib in TRK fusion-positive cancers in adults and children. N Engl J Med 378(8): 731-739.

14. Gounder MM, Stacchiotti S, Schöffski P, Attia S, Italiano A, et al. (2017) Phase 2 multicenter study of the EZH2 inhibitor tazemetostat in adults with INI1 negative epithelioid sarcoma (NCT02601950). J Clin Oncol 
35(suppl abstract 11058).

15. Paoluzzi L, Cacavio A, Ghesani M, Karambelkar A, Rapkiewicz A, et al.
(2016) Response to anti-PD1 therapy with nivolumab in metastatic sarcomas. Clin Sarcoma Res 6: 24.

\section{Your next submission with Juniper Publishers will reach you the below assets}

- Quality Editorial service

- Swift Peer Review

- Reprints availability

- E-prints Service

- Manuscript Podcast for convenient understanding

- Global attainment for your research

- Manuscript accessibility in different formats ( Pdf, E-pub, Full Text, Audio)

- Unceasing customer service

Track the below URL for one-step submission https://juniperpublishers.com/online-submission.php 